\title{
Modeling Population Growth of Pyrenean Chamois (Rupicapra p. pyrenaica) by Using P-Systems
}

\author{
Maria Angels Colomer ${ }^{1}$, Santiago Lavín ${ }^{2}$, Ignasi Marco ${ }^{2}$, Antoni Margalida ${ }^{3}$, \\ Ignacio Pérez-Hurtado ${ }^{4}$, Mario J. Pérez-Jiménez ${ }^{4}$, Delfí Sanuy ${ }^{5}$, \\ Emmanuel Serrano $^{2}$, and Luis Valencia-Cabrera ${ }^{4}$ \\ 1 Dpt. of Mathematics, University of Lleida \\ Av. Alcalde Rovira Roure, 191. 25198 Lleida, Spain \\ colomer@matematica.udl.es \\ 2 Servei d'Ecopatologia de Fauna Salvatge (SEFaS), Departament de Medicina i \\ Cirurgia Animals, Universitat Autònoma de Barcelona (UAB), \\ E-08193-Bellaterra (Barcelona), Spain \\ \{Santiago.Lavin, Ignasi .Marco, Emmanuel.Serrano\}@uab.cat \\ 3 Bearded Vulture Study \& Protection Group \\ Apdo. 43 E-25520 El Pont de Suert (Lleida), Spain \\ margalida@inf .entorno.es \\ 4 Research Group on Natural Computing \\ Dpt. of Computer Science and Artificial Intelligence, University of Sevilla \\ Avda. Reina Mercedes s/n. 41012 Sevilla, Spain \\ \{perezh, marper, lvalencia\}@us.es \\ 5 Dpt. of Animal Production, University of Lleida \\ Av. Alcalde Rovira Roure, 191. 25198 Lleida, Spain \\ dsanuy@prodan.udl.cat
}

\begin{abstract}
P systems provide a high level computational modeling framework which integrates the structural and dynamic aspects of ecosystems in a comprehensive and relevant way. In previous works,several ecosystems modeled by using $\mathrm{P}$ systems were presented. The good results obtained encourage us to study new ecosystems such as the one presented in this paper. Pyrenean Chamois (Rupicapra p. pyrenaica) is an ungulate species inhabiting the Catalan Pyrenees. In recent years, several diseases have caused a drastic decrease in the number of individuals. Since they provide significant economic contributions in the area and constitutes an important food resource for obligate and facultative scavengers, it is very interesting to provide a model in order to facilitate the management of their ecosystems.
\end{abstract}

\section{Introduction}

Modeling a biological system is usually very complicated because each biological process involves a large number of factors interacting with each other. Therefore the most common solution is to define it a scenario in which the number of variables and interactions between variables is very limited. 
Most of the existing models for the study of population dynamics are based on differential equations (ODEs), but this approach has some drawbacks. When the number of species in a model is greater than two, the equations system proposed is so complex that it is usually solved using numerical methods. Besides, improvements on the performance of the models are generally obtained by the addition of ingredients, which in the case of ODEs means that the whole modeling process needs to start from scratch.

Computer models based on P system offer significant advantages: modularity, parallelism, and no limitation on the number of interrelated variables that evolve parallely. These properties make them very attractive for modeling complex ecosystems.

Each ecosystem has its own important peculiarities, thus trying to design an "universal" ecosystem model is not a good approach. The model should be adapted taking into account if a protected and endangered species is being studied, or if the ecosystem deals with an invasive species, or simply an endemic area.

Nevertheless, there are some aspects common to most ecosystems such as:

- They contain a large number of individuals and species.

- The life cycles of species inhabiting the ecosystem display several basic processes such as: feeding, growth, reproduction and death.

- These processes are repeated annually.

- The evolution often depends on the environment: weather, soil, vegetation, etc.

- The natural dynamics suffers modifications due to human activities.

These common features yield some requisites for the model from a computational point of view: many processes take place simultaneously, there is cooperation between individuals and elements of the ecosystem, partial synchronization among the dynamic evolution sub-ecosystems (for example, there could be adverse weather conditions some year, and this does not affect a single subecosystem, but has a global influence on the entire ecosystem), situations need to be restored annually.

These considerations lead to the definition of an appropriate modeling semantic context for the $\mathrm{P}$ system. In particular, a precise semantics of the multienvironment functional probabilistic $\mathrm{P}$ system with active membranes has been used to model two real ecosystems: One based on scavenger birds in the Catalan Pyrenees (Spain) [1] and another one based on the zebra mussel (Dreissena polymorpha) in the Ribarroja reservoir (Spain) [2. In the first case, an endangered species is modeled and the purpose of the obtained model is the study of the evolution of the ecosystem under different scenarios to make the most appropriate management decisions for the conservation of species. The second study case corresponds to a completely different situation, the zebra mussel is an exotic species that has shown an excellent adaptation after its introduction in the reservoir. Its uncontrolled reproduction causes significant economic and ecological damage. 
In both cases we have designed a simulator to validate the results so that managers have now two tools enabling them to perform virtual experiments under different conditions.

To show that the proposed modeling framework enables the study of a wide range of ecosystems, this paper presents a model for the study of the Pyrenean Chamois dynamics in the Catalan Pyrenees (Spain). It is an emblematic species that was in danger of extinction a few years ago and have an important role on the chain food of this area. Aquila chrysaetos

\section{Pyrenean Chamois}

It is a small ungulate living in the Pyrenees of great interest, not only from a hunting standpoint, but also naturalistic and touristic. At present the existing population in the Pyrenees is estimated to be 53,000 individuals. The status of the species has not always been so favorable, for example, in the late 60 s the population decreased down to the edge of extinction due to indiscriminate hunting. Fortunately, National Hunting Reserves managed by the regional administration were created in order to save the species.

This species has no major predators in the Pyrenees, except for the brown bear (Ursus arctos), and the golden eagle (Aquila chrysaetos). However it is a species with a small growth rate compared with other species of ungulates. In recent years, the population has suffered from several epidemics of infectious keratoconjunctivitis and, more recently from a new disease associated to a Border Disease Virus (Genus Pestivirus, Familiy Flaviviridae) has affected some Pyrenean chamois populations. However, only the second one has been responsible for local population decreases greater than $80 \%$ (Marco et al 2009). The Pyrenean chamois has a life expectancy of 20 years, though the mortality rate is high for animals older than 11 years. In early ages, the mortality rate ranges from $40 \%$ to $50 \%$, while it is around $10 \%$ for adults younger than 11 years. At the age of two, they reach the reproductive age, and approximately $75 \%$ of females mate once a year generally producing one single descendant.

The disease associated to a Pestivirus is having a very important impact on a social and economic scale in the Pyrenees. The media have been blare of different epidemics occurring, being a reflection of the concerns of local communities, the Government of Catalonia, ranchers, hiking groups, conservationists and hunters. The suspension of Pyrenean chamois hunting in the affected areas has led to major loss of economic income. This loss is due not only to the lack of direct income through payment of hunting licences, but also by the disappearance of the indirect income (ecoturism) that hunters and their guests bring out. Last but not least, we must highlight the considerable ecological impact of the sudden disappearance of this herbivore in the affected areas. Despite the detailed studies being carried out currently, the resulting consequences in the ecosystem are still unclear.

In Spain, Pestivirus infection of the species is up to now affecting only the region of Catalonia. However, it is possible that the process spreads to other regions. The process also affects the French Pyrenees sector, which borders the affected Catalan area [6]. 
In the Catalan Pyrenees there are 5 main protected areas where Pyrenean chamois live in herds, figure 1, We assume that it is unlikely that this species moves between areas and hence, wherever there is a lack of resources the animals die.

In this work we aim to present a model to simulate the evolution of the Pyrenean Chamois populations in the Catalan Pyrenees.

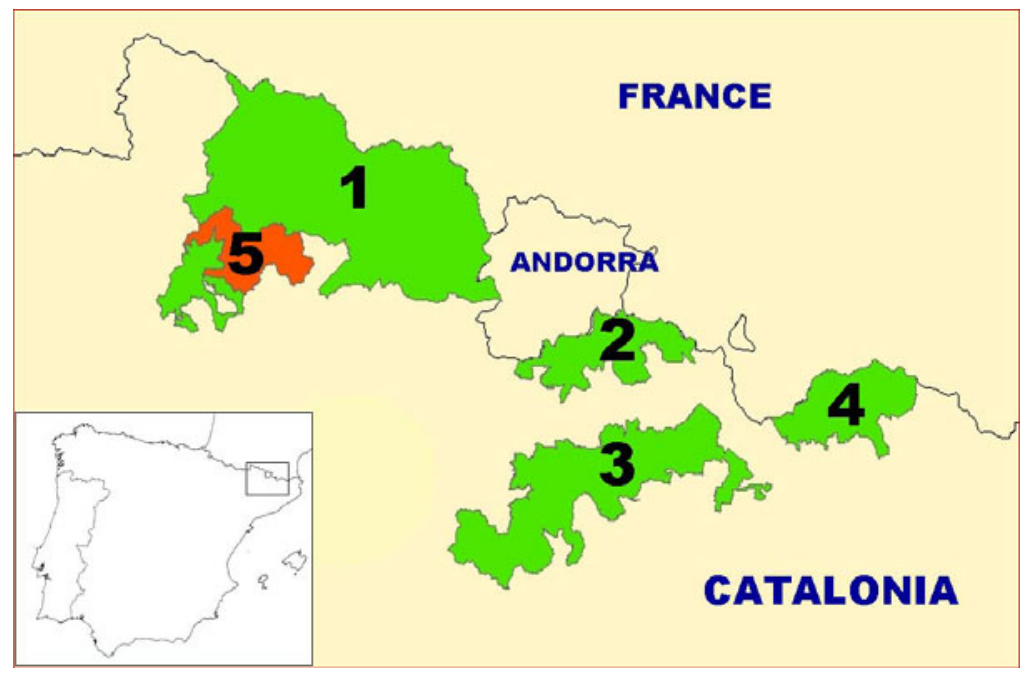

Fig. 1. Study area in the Catalan Pyrenees. Area 1: National Reservoir of hunting in l'Alt Pallars-Aran. Area 2: RNC Cerdanya-Alt Urgell. Area 3: RNC Cadí. Area 4: RNC Freser-Setcases. Area 5: Parc Nacional, not include in the study.

\section{A P System Based Modeling Framework}

It will define the variant of P-Sytem to be used for modeling the Pyrenean Chamois.

Definition 1. A multienvironment functional probabilistic $P$ system with active membranes of degree $(q, m)$ with $q \geq 1, m \geq 1$, taking $T$ time units, $T \geq 1$, is a tuple

$\left(G, \Gamma, \Sigma, R_{E}, \Pi,\left\{f_{r, j}: r \in R_{\Pi}, 1 \leq j \leq m\right\},\left\{\mathcal{M}_{i, j}: 0 \leq i \leq q-1,1 \leq j \leq m\right\}\right)$

where:

- $G=(V, S)$ is a directed graph such that $(x, x) \in S$, for each $x \in V$. Let $V=\left\{e_{1}, \ldots, e_{m}\right\}$ whose elements are called environments;

- $\Gamma$ is the working alphabet and $\Sigma \varsubsetneqq \Gamma$ is an alphabet representing the objects that can be present in the environments; 
- $R_{E}$ is a finite set of communication rules between environments of the form

$$
(x)_{e_{j}} \stackrel{p_{\left(x, j, j, j_{1}, \ldots, j_{h}\right)}}{\longrightarrow}\left(y_{1}\right)_{e_{j_{1}}} \ldots\left(y_{h}\right)_{e_{j_{h}}}
$$

where $x, y_{1}, \ldots, y_{h} \in \Sigma,\left(e_{j}, e_{j_{l}}\right) \in S(l=1, \ldots, h)$ and $p_{\left(x, j, j_{1}, \ldots, j_{h}\right)}(t) \in$ $[0,1]$, for each $t=1, \ldots, T$. If $p_{\left(x, j, j_{1}, \ldots, j_{h}\right)}(t)=1$, for each $t$, then we omit the probabilistic function. These rules verify the following:

$\star$ for each $e_{j}$ and for each $x$, the sum of functions associated with the rules from $R_{E}$ whose left-hand side is $(x)_{e_{j}}$ coincide with the constant function equal to 1 .

$-\Pi=\left(\Gamma, \mu, R_{\Pi}\right)$ where

- $\mu$ is a membrane structure consisting of $q$ membranes, with the membranes injectively labeled with $0, \ldots, q-1$. The skin membrane is labeled with 0 . We also associate electrical charges from the set $\{0,+,-\}$ with membranes; and

- $R_{\Pi}$ is a finite set of evolution rules of the form $r: u[v]_{i}^{\alpha} \rightarrow u^{\prime}\left[v^{\prime}\right]_{i}^{\alpha^{\prime}}$ where $u, v, u^{\prime}, v^{\prime} \in M(\Gamma), i \in\{0,1, \ldots, q-1\}$, and $\alpha, \alpha^{\prime} \in\{0,+,-\}$;

- For each $r \in R_{\Pi}$ and for each $j, 1 \leq j \leq m, f_{r, j}$ is a computable function whose domain is $\{1,2, \ldots, T\}$ and its range is contained in $[0,1]$, verifying the following:

$\star$ For each $u, v \in M(\Gamma), i \in\{0, \ldots, q-1\}$ and $\alpha \in\{0,+,-\}$, if $r_{1}, \ldots, r_{z}$ are the rules from $R_{\Pi}$ whose left-hand side is $u[v]_{i}^{\alpha}$, then $\sum_{j=1}^{z} f_{r, j}(t)=$ 1 , for each $t, 1 \leq t \leq T$.

- For each $j(1 \leq j \leq m), \mathcal{M}_{0, j}, \ldots, \mathcal{M}_{q-1, j}$ are strings over $\Gamma$, describing the multisets of objects initially placed in the $q$ regions of $\mu$.

A multienvironment probabilistic functional extended $\mathrm{P}$ system with active membranes of degree $(q, m)$ taking $T$ time units

$\left(G, \Gamma, \Sigma, R_{E}, \Pi,\left\{f_{r, j}: r \in R_{\Pi}, 1 \leq j \leq m\right\},\left\{\mathcal{M}_{i, j}: 0 \leq i \leq q-1,1 \leq j \leq m\right\}\right)$

can be viewed as a set of $m$ environments $e_{1}, \ldots, e_{m}$ linked between them by the arcs from the directed graph $G$. Each environment $e_{j}$ contains a functional probabilistic $\mathrm{P}$ system with active membranes of degree $q$, each of them with the same skeleton, $\Pi$, and such that $\mathcal{M}_{0 j}, \ldots \mathcal{M}_{q-1, j}$ describe their initial multisets.

When a communication rule between environments

$$
(x)_{e_{j}} \stackrel{p\left(x, j, j_{1}, \ldots, j_{h}\right)}{\longrightarrow}\left(y_{1}\right)_{e_{j_{1}}} \ldots\left(y_{h}\right)_{e_{j_{h}}}
$$

is applied, object $x$ pass from $e_{j}$ to $e_{j_{1}}, \ldots, e_{j_{h}}$ possibly modified into objects $y_{1}, \ldots, y_{h}$, respectively. At any moment $t, 1 \leq t \leq T$, in which an object $x$ is in environment $e_{j}$, one and only one rule will be applied according to its probability which is given by $p_{\left(x, j, j_{1}, \ldots, j_{h}\right)}(t)$.

We assume that a global clock exists, marking the time for the whole system (for its compartments), that is, all membranes and the application of all rules are synchronized.

The tuple of multisets of objects present at any moment in the $m$ environments and at each of the regions of the $\mathrm{P}$ systems located within them, and the 
polarizations of the membranes in each $\mathrm{P}$ system, constitutes a configuration of the system at that moment. At the initial configuration of the system we assume that all environments are empty and all membranes have a neutral polarization.

The $\mathrm{P}$ system can pass from one configuration to another by using the rules from $R=R_{E} \cup \bigcup_{j=1}^{m} R_{\Pi_{j}}$ as follows: at each transition step, the rules to be applied are selected according to the probabilities assigned to them, and all applicable rules are simultaneously applied and all occurrences of the left-hand side of the rules are consumed, as usual.

\section{Model}

Pyrenean chamois is one of the species that were considered in the ecosystem modeled in 1]. However, that paper focuses mainly on the processes of feeding, reproduction, and mortality, including also that migration to other areas may occur in the event of resources shortage. The possibility that the species could be affected by disease was not taken into account, neither the fact that some biological parameters depend on the weather.

Taking advantage of the modularity of the $\mathrm{P}$ system, in order to cover these new aspects it suffices to modify the schema of the model presented in [1] by adding two modules, weather and disease module. In this work we have divided the mortality module in two, natural and hunter module. Besides, modules related to exchange between environments will be removed, since Pyrenean chamois do not migrate when there are not enough resources (as mentioned in section 2).

We present a preliminary study of the dynamics of Pyrenean Chamois, taking into account the following considerations:

- There are four separated areas in the Catalan Pyrenees were the Pyrenean chamois lives.

- Weather conditions, especially in winter (particularly the thickness of the snow layer), influence the values of biological parameters of the Pyrenean chamois species 4 .

- Causes of death for this species include: natural death, hunting and disease. Only Pestivirus infection will be taken into account, while other diseases of importance that are known to affect the species will not be considered yet.

- The possibility of introducing more species in the model remains open. Note that in the same geographical space other wild and domestic ungulates may coexist in some cases, and this is worth studying especially if these species are competing for food with the parameters of the species.

The algorithmic scheme of the proposed model is shown in figure 2. The algorithm has been sequenced, but all animals evolve in parallel. The processes to be modeled will be the weather conditions (snow), reproduction, regulation of density, food, natural mortality, hunting mortality and mortality due to a disease. In order to model these processes for each species it is needed some biological, geographical and human factors, that is shown in Table1. 


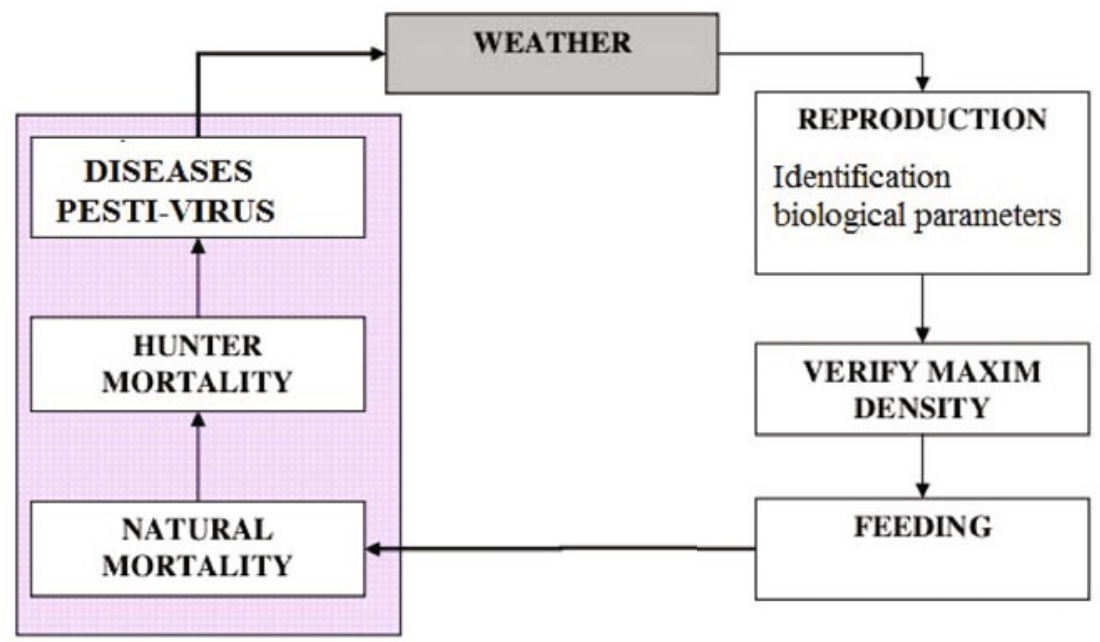

Fig. 2. Scheme model of the Pyrenean chamois model

The proposed model consists of a multienvironment functional probabilistic $\mathrm{P}$ system with active membranes of degree $(4,11)$, taking $T$ times units

$$
\left(G, \Gamma, \Sigma, R_{E}, \Pi,\left\{f_{r, \nu}: r \in R_{\Pi}, 1 \leq \nu \leq 4\right\},\left\{M_{i, \nu}: 0 \leq i \leq 10,1 \leq \nu \leq 4\right\}\right)
$$

where:

- The graph of the system is $G=(V, S)$, where $V=\left\{e_{1}, \ldots, e_{4}\right\}$ is the set of nodes called environments, and $S=\left\{\left(e_{1}, e_{i}\right): 1 \leq i \leq 4\right\}$.

- The working alphabet is

$$
\begin{aligned}
\Gamma= & \left\{X_{j y}, Y_{j y}, Y_{j y}^{\prime}, Y_{j y}^{\prime \prime}, Z_{j y}, V_{j y}, W_{j y}: 0 \leq j \leq g_{3}, 1 \leq y \leq T\right\} \cup \\
& \left\{a, c, d, e, t, h, d_{1} F, D, S, N\right\} \cup\left\{t_{i}: 1 \leq i \leq 3\right\} \cup\left\{G_{i}: 4 \leq i \leq 10\right\} \cup \\
& \left\{R_{i}: 0 \leq i \leq 7\right\}
\end{aligned}
$$

The objects $X, Y, Y^{\prime}, Y^{\prime \prime}, Z, V$ and $W$ are associated with animals in different states, index $j$ represents the age of the animal and index $y$ represents the moment of the simulation. The $t$ are objects associated with the weather. $F$ is an object that allows the generation of food in the form of grass. $G_{i}$ are objects associated with the production of grass in the month $i$. The objects $D, c$, and $e$ are used to control the density of animals of each species. The objects $h_{1}$ and $h$ are used in order to know the state of Pesti-virus. The objects $S$ and $N$ indicate presence or absence of the disease, respectively, and finally there is the counter $R$ that will allow us to synchronize the $\mathrm{P}$ System.

- The environment alphabet is

$$
\Sigma=\left\{t, t_{i}: 1 \leq i \leq 10\right\}
$$


Table 1. Biological and geographical information $(i$ month, $\nu$ area, $l$ Snow thickness category)

\begin{tabular}{|l|c|}
\hline Biological & Parameter \\
\hline \hline Age at which they are considered adults & $g_{0}$ \\
\hline Age at which they begin to be fertile & $g_{2}$ \\
\hline Age at which they cease to be fertile & $g_{3}$ \\
\hline Life expectancy & $k_{1}$ \\
\hline Proportion of females in the population (as per 1) & $k_{2}$ \\
\hline Fertility rate (as per 1) & $k_{3}$ \\
\hline Number of descendants per female & $m_{1}$ \\
\hline Rate of natural mortality on young animals (as per 1) & $m_{2}$ \\
\hline Rate of natural mortality on adult animals (as per 1) & Parameter \\
\hline Amount of grass consumed per month and animal & $m \leq 10$ \\
\hline \hline Geographical & $m s_{\nu}, 1 \leq \nu \leq 4$ \\
\hline \hline Amount of grass produced per month & $m d_{\nu}, 1 \leq \nu \leq 4$ \\
\hline Probability of having the disease & $d 1_{\nu}, 1 \leq \nu \leq 4$ \\
\hline Probability of dying from a disease & $d 2_{\nu}, 1 \leq \nu \leq 4$ \\
\hline Maximum density of the ecosystem & Parameter \\
\hline $\begin{array}{l}\text { Number of animals that survive after reaching } \\
\text { the maximum density }\end{array}$ & $h 1_{\nu}, 1 \leq \nu \leq 4$ \\
\hline \hline Human factors & $h 2_{\nu}, 1 \leq \nu \leq 4$ \\
\hline \hline Young animals hunted & \\
\hline Adult animals hunted & \\
\hline
\end{tabular}

- The purpose of the set $R_{E}$ of environment rules is to select the weather conditions for the year, and to distribute this information to all environments. This is done because there are some biological parameters that vary depending on weather conditions. In particular, we are able to simulate the snow thickness.

$$
\begin{aligned}
& r_{e_{1}} \equiv(t)_{e_{1}} \stackrel{1 / 10}{\longrightarrow}\left(t_{i}\right)_{e_{1}}\left(t_{i}\right)_{e_{2}}\left(t_{i}\right)_{e_{3}}\left(t_{i}\right)_{e_{4}} 1 \leq i \leq 10 \\
& r_{e_{2}} \equiv(t)_{e_{k}} \rightarrow(\#)_{e_{k}}, 1<k \leq 4
\end{aligned}
$$

$-\Pi=\left(\Gamma, \mu, R_{\Pi}\right)$ is the skeleton of a functional probabilistic $\mathrm{P}$ system with active membranes of degree 11 , whose membrane structure is $\mu=\left[[]_{1}[]_{2} \ldots\right.$ []$\left._{10}\right]_{0}$.

The set $R_{\Pi}$ of rules of the system is the following (where the probabilistic constants associated with the rules have been incorporated):

* Preparation of the system to start a cycle.

$r_{1} \equiv t_{i}[]_{0}^{0} \rightarrow\left[t_{i}\right]_{0}^{0}, 1 \leq i \leq 10$.

$r_{2} \equiv t_{i}[]_{i}^{0} \rightarrow[t]_{i}^{-}, 1 \leq i \leq 10$.

After applying the environment rules, $r_{e_{1}}$ object $t_{i}$ enters from the environment carrying the information about the climatic condition of the next year to be simulated. 
Each of the inner membranes labeled with 1, 2 or 10, stores information on biological parameters for each one of the then different climatic scenarios that the model envisages. The objects associated with animals should then enter the same membrane as object $t_{i}$.

$r_{3} \equiv X_{j, y}[]_{k}^{-} \rightarrow\left[X_{j, y}\right]_{k}^{0},\left\{\begin{array}{l}1 \leq j \leq g_{3} \\ 1 \leq y \leq T \\ 1 \leq k \leq 10\end{array}\right.$

Each geographic area in which the species lives has a monthly production of food (grass).

$r_{4} \equiv\left(F[]_{k}^{-} \rightarrow\left[G_{4}^{\alpha_{4}(\nu)}, \ldots, G_{10}^{\alpha_{10}(\nu)}\right]_{k}^{0}\right)_{e_{\nu}},\left\{\begin{array}{l}1 \leq k \leq 10 \\ 1 \leq \nu \leq 4\end{array}\right.$

where $\alpha_{j}(\nu)$ corresponds to the amount of grass produced in the area $\nu$ for the month $j$.

When the pesti-virus appears in an area, object $h$ is produced. It will be present in the following configurations.

$r_{5} \equiv h[]_{k}^{-} \rightarrow[h]_{k}^{0}, 1 \leq k \leq 10$

The amount of animals in the ecosystem should be controled so that it can not exceed a maximum load. This operation is performed by the objects $a$.

$r_{6} \equiv\left(c[]_{k}^{-} \rightarrow\left[a^{0.9 d 1_{\nu}} e^{0.2 d 1_{\nu}}\right]_{k}^{0}\right)_{e_{\nu}},\left\{\begin{array}{l}1 \leq k \leq 10 \\ 1 \leq \nu \leq 4\end{array}\right.$

The following rules simulate the presence or absence of disease.

$$
\begin{aligned}
& r_{7} \equiv d[]_{k}^{-} \rightarrow[d]_{k}^{0}, 1 \leq k \leq 10 . \\
& r_{8} \equiv\left[d h \rightarrow d_{1}\right]_{k}^{0}, 1 \leq k \leq 10 . ; \\
& r_{9} \equiv\left(\left[d_{1}-\stackrel{m s_{\nu}}{\longrightarrow} S\right]_{k}^{0}\right)_{e_{\nu}},\left\{\begin{array}{l}
1 \leq k \leq 10, \\
1 \leq \nu \leq 4 .
\end{array}\right. \\
& r_{10} \equiv\left(\left[d_{1} \stackrel{1-m s_{\nu}}{\longrightarrow} N\right]_{k}^{0}\right)_{e_{\nu}}, \quad\left\{\begin{array}{l}
1 \leq k \leq 10, \\
1 \leq \nu \leq 4 .
\end{array}\right.
\end{aligned}
$$

Then we have counter $R_{i}$ that will allow us to synchronize the $\mathrm{P}$ system

$$
r_{11} \equiv R_{0}[]_{k}^{-} \rightarrow\left[R_{0}\right]_{k}^{0}, 1 \leq k \leq 10 \text {. }
$$$$
r_{12} \equiv\left[R_{i} \rightarrow R_{i+1}\right]_{k}^{0},\left\{\begin{array}{l}
0 \leq i \leq 4, \\
1 \leq k \leq 10
\end{array}\right.
$$

Finally, we introduce some randomness in the density control

$$
\begin{aligned}
& r_{13} \equiv[e \stackrel{0.5}{\longrightarrow} a]_{k}^{0}, 1 \leq k \leq 10 . \\
& r_{14} \equiv[e \stackrel{0.5}{\longrightarrow} \#]_{k}^{0}, 1 \leq k \leq 10 .
\end{aligned}
$$


* Reproduction rules

Males of childbearing age

$$
r_{15} \equiv\left[X_{j, y} \stackrel{1-k_{1}}{\longrightarrow} Y_{j, y} D\right]_{k}^{0},\left\{\begin{array}{l}
g_{1} \leq j<g_{2}, \\
1 \leq y \leq T \\
1 \leq k \leq 10
\end{array}\right.
$$

Females of childbearing age that reproduce

$$
r_{16} \equiv\left[X_{j, y} \stackrel{{ }^{k 1 \cdot k 2_{l}} \longrightarrow}{\longrightarrow} Y_{j, y} Y_{0, y}^{k_{3}} D^{k_{3}+1}\right]_{k}^{0},\left\{\begin{array}{l}
g_{1} \leq j<g_{2}, \\
1 \leq y \leq T \\
1 \leq k \leq 10
\end{array}\right.
$$

Females of childbearing age that do not reproduce

$$
r_{17} \equiv\left[X_{j, y} \stackrel{k 1 \cdot\left(1-k 2_{l}\right)}{\longrightarrow} Y_{j, y} D\right]_{k}^{0},\left\{\begin{array}{l}
g_{1} \leq j<g_{2}, \\
1 \leq y \leq T \\
1 \leq k \leq 10
\end{array}\right.
$$

Animals that are not fertile

$$
r_{18} \equiv\left[X_{j, y} \rightarrow Y_{j, y} D\right]_{k}^{0},\left\{\begin{array}{l}
g_{2} \leq j \leq g_{3} \\
1 \leq y \leq T \\
1 \leq k \leq 10
\end{array}\right.
$$

Young animals that do not reproduce

$$
r_{19} \equiv\left[X_{j, y} \rightarrow Y_{j, y} D\right]_{k}^{0},\left\{\begin{array}{l}
1 \leq j<g_{2}, \\
1 \leq y \leq T \\
1 \leq k \leq 10
\end{array}\right.
$$

* Density rules

Checking if the maximum density has been reached

$$
\begin{aligned}
& \left.r_{20} \equiv\left(\left[D^{d 1_{\nu}} a^{d 1_{\nu}-d 2_{\nu}}\right]_{k}^{0} \rightarrow\left[h_{0}\right]\right]_{k}^{0}\right)_{e_{\nu}},\left\{\begin{array}{l}
1 \leq k \leq 10, \\
1 \leq \nu \leq 4 .
\end{array}\right. \\
& r_{21} \equiv\left[d, h_{0}\right]_{k}^{0} \rightarrow\left[d_{0}\right]_{k}^{0}, 1 \leq k \leq 10 .
\end{aligned}
$$

Transformation of objects that represent animals

$$
r_{22} \equiv\left[Y_{j, y} \rightarrow Y_{j, y}^{\prime}\right]_{k}^{0},\left\{\begin{array}{l}
0 \leq j \leq g_{3} \\
1 \leq y \leq T \\
1 \leq k \leq 10
\end{array}\right.
$$

\section{* Feeding rules}

$r_{23} \equiv\left[Y_{j, y}^{\prime} a G_{4}^{\beta_{4}} G_{5}^{\beta_{5}} G_{6}^{\beta_{6}} G_{7}^{\beta_{7}} G_{8}^{\beta_{8}} G_{9}^{\beta_{9}} G_{10}^{\beta_{10}} \rightarrow Z_{j, y}\right]_{k}^{0},\left\{\begin{array}{l}0 \leq j \leq g_{3}, \\ 1 \leq y \leq T \\ 1 \leq k \leq 10 .\end{array}\right.$

where $\beta_{i}$ represents the need of food in month $i$. 
* Natural mortality rules

Young animals that survive

$$
r_{24} \equiv\left(\left[Z_{j, y} \stackrel{1-m 1_{k, \nu}}{\longrightarrow} V_{j, y}\right]_{k}^{0}\right)_{e_{\nu}},\left\{\begin{array}{l}
0 \leq j<g_{0} \\
1 \leq y \leq T \\
1 \leq k \leq 10 \\
1 \leq \nu \leq 4
\end{array}\right.
$$

Young animals that leave the ecosystem or die

$$
r_{25} \equiv\left(\left[Z_{j, y} \stackrel{m 1_{k, \nu}}{\longrightarrow} \#\right]_{k}^{0}\right)_{e_{\nu}},\left\{\begin{array}{l}
0 \leq j<g_{0}, \\
1 \leq y \leq T, \\
1 \leq k \leq 10 \\
1 \leq \nu \leq 4
\end{array}\right.
$$

Adult animals that survive

$$
r_{26} \equiv\left[Z_{j, y} \stackrel{1-m 2}{\longrightarrow} V_{j, y}\right]_{k}^{0},\left\{\begin{array}{l}
g_{0} \leq j<g_{3}, \\
1 \leq y \leq T, \\
1 \leq k \leq 10
\end{array}\right.
$$

Adult animals that die

$r_{27} \equiv\left[Z_{j, y} \stackrel{m 2}{\longrightarrow} \#\right]_{k}^{0},\left\{\begin{array}{l}g_{0} \leq j<g_{3}, \\ 1 \leq y \leq T \\ 1 \leq k \leq 10\end{array}\right.$

Animals that reach the maximum age of the species

$r_{28} \equiv\left[Y_{g_{3}, y} \rightarrow \#\right]_{k}^{0},\left\{\begin{array}{l}1 \leq y \leq T, \\ 1 \leq k \leq 10 .\end{array}\right.$

* Hunting mortality

Young animals that survive hunting

$$
r_{29} \equiv\left(\left[V_{j, y} \stackrel{1-h 1_{\nu}}{\longrightarrow} W_{j, y}\right]_{k}^{0}\right)_{e_{\nu}},\left\{\begin{array}{l}
0 \leq j<g_{0} \\
1 \leq y \leq T \\
1 \leq k \leq 10 \\
1 \leq \nu \leq 4
\end{array}\right.
$$

Young animals that are hunted

$$
r_{30} \equiv\left(\left[V_{j, y} \stackrel{h 1_{\nu}}{\longrightarrow} \#\right]_{k}^{0}\right)_{e_{\nu}},\left\{\begin{array}{l}
0 \leq j<g_{0} \\
1 \leq y \leq T \\
1 \leq k \leq 10 \\
1 \leq \nu \leq 4
\end{array}\right.
$$

Adult animals that survive hunting

$$
r_{31} \equiv\left(\left[V_{j, y} \stackrel{1-h 2_{\nu}}{\longrightarrow} W_{j, y}\right]_{k}^{0}\right)_{e_{\nu}},\left\{\begin{array}{l}
g_{0} \leq j<g_{3} \\
1 \leq y \leq T \\
1 \leq k \leq 10 \\
1 \leq \nu \leq 4
\end{array}\right.
$$


Adult animals that are hunted

$$
r_{32} \equiv\left(\left[V_{j, y} \stackrel{h 2_{\nu}}{\longrightarrow} \#\right]_{k}^{0}\right)_{e_{\nu}},\left\{\begin{array}{l}
g_{0} \leq j<g_{3} \\
1 \leq y \leq T \\
1 \leq k \leq 10 \\
1 \leq \nu \leq 4
\end{array}\right.
$$

* Disease mortality

$$
\begin{aligned}
& r_{33} \equiv\left[R_{5} S\right]_{k}^{0} \rightarrow\left[R_{6} h\right]_{k}^{-}, 1 \leq k \leq 10 . \\
& r_{34} \equiv\left[R_{5} N \rightarrow R_{6} h\right]_{k}^{0}, 1 \leq k \leq 10 . \\
& r_{35} \equiv\left[R_{5} d_{0} \rightarrow R_{6} h\right]_{k}^{0}, 1 \leq k \leq 10 . \\
& r_{36} \equiv\left[R_{5} d \rightarrow R_{6}\right]_{k}^{0}, 1 \leq k \leq 10 . \\
& r_{37} \equiv\left[R_{6}\right]_{k}^{-} \rightarrow[\#]_{k}^{+}, 1 \leq k \leq 10 . \\
& r_{38} \equiv\left[R_{6}\right]_{k}^{0} \rightarrow[\#]_{k}^{+}, 1 \leq k \leq 10 . \\
& r_{39} \equiv\left(\left[W_{j, y}\right]_{k}^{-} \stackrel{m d_{\nu}}{\longrightarrow}[\#]_{k}^{+}\right)_{e_{\nu}},\left\{\begin{array}{l}
0 \leq j<g_{3}, \\
1 \leq y \leq T, \\
1 \leq k \leq 10 \\
1 \leq \nu \leq 4
\end{array}\right. \\
& r_{40} \equiv\left(\left[W_{j, y}\right]_{k}^{-} \stackrel{1-m d_{\nu}}{\longrightarrow}\left[W_{j, y}\right]_{k}^{+}\right)_{e_{\nu}},\left\{\begin{array}{l}
0 \leq j<g_{3} \\
1 \leq y \leq T \\
1 \leq k \leq 10 \\
1 \leq \nu \leq 4
\end{array}\right.
\end{aligned}
$$

* Updating rules

$$
\begin{aligned}
& r_{41} \equiv\left[W_{j, y}\right]_{k}^{+} \rightarrow X_{j+1, y+1}[]_{k}^{0},\left\{\begin{array}{l}
0 \leq j<g_{3}, \\
1 \leq y \leq T, \\
1 \leq k \leq 10
\end{array}\right. \\
& r_{42} \equiv\left[Y_{j, y}^{\prime}\right]_{k}^{+} \rightarrow[\#]_{k}^{0}, \quad\left\{\begin{array}{l}
0 \leq j<g_{3}, \\
1 \leq y \leq T, \\
1 \leq k \leq 10 .
\end{array}\right. \\
& r_{43} \equiv[t]_{k}^{+} \rightarrow R_{0}, F, t, c, d[]_{k}^{0}, 1 \leq k \leq 10 . \\
& r_{44} \equiv[h]_{k}^{+} \rightarrow h[]_{k}^{0}, 1 \leq k \leq 10 . \\
& r_{45} \equiv[a]_{k}^{+} \rightarrow[\#]_{k}^{0}, 1 \leq k \leq 10 . \\
& r_{46} \equiv\left[G_{i}\right]_{l}^{+} \rightarrow[\#]_{k}^{0}, \quad\left\{\begin{array}{l}
4 \leq i \leq 10 \\
1 \leq k \leq 10 .
\end{array}\right. \\
& r_{47} \equiv[t]_{0}^{0} \rightarrow t[]_{0}^{0}
\end{aligned}
$$

- $\mathcal{M}_{0, \nu}, \ldots \mathcal{M}_{10, \nu}(1 \leq \nu \leq 4)$ are strings over $\Gamma$ which describe the initial multiset of objects located in the regions of $\mu$.

$$
\begin{aligned}
& \mathcal{M}_{0, \nu}=\left\{F, R_{0}, c, d\right\} \cup\left\{X_{j, 1}^{q_{\nu, j}}: 1 \leq \nu \leq 4,1 \leq j \leq g_{3}\right\}, \quad \text { for } 1 \leq \nu \leq 4 \\
& \mathcal{M}_{i, \nu}=\emptyset, \text { for } 1 \leq i \leq 10 \text { and } 1 \leq \nu \leq 4
\end{aligned}
$$




\section{A Software Tool for Simulation}

A software tool under GNU GPL license 8] for simulating $\mathrm{P}$ systems modeling ecosystems was presented in 2 . The simulation of two real and relevant ecosystems has been achieved by using this software tool [2. One of them is related to an endangered species (the bearded vulture) and the other one is related to an exotic and invasive species (the zebra mussel). For each one, an ad hoc graphic user interface (GUI) has been developed in order to configure the initial parameters of the ecosystem and collect the results of the simulation by means of tables and graphics. The simulation core that has been used is based on P-Lingua [59] and pLinguaCore [510]. The software tool allows two different types of users: the first one is the designer user, who is the responsible for defining, debugging and validating the model for the ecosystem; and the second one is the end-user, who is the final user of the software tool and he/she uses it for carrying out virtual experiments over the ecosystem.

One of the main problems of the software tool is the need to design, develop and maintain several different graphic user interfaces. In this paper, a new software tool, MeCoSim [7], has been used. MeCoSim (Membrane Computing Simulator) allows the same functionality as its predecessor, and besides the designer user is provided with an easy-to-use method for creating new ad $h o c$ GUIs for specific ecosystem models, by means of the definition in data bases. In this sense, the development of the GUI in Java Swing (or other programming languages) has been avoided, delegating this process on the designer user.

To summarize, MeCoSim offers to the users (designer and end-user) a highly customizable simulators generator to apply simulation algorithms for $\mathrm{P}$ systems modelling several scenarios under study. Thus, MeCoSim is a final product that avoid the necessity of ad-hoc GUI development per each scenario, introducing enough flexibility to permit the designer user to generate a simulator adapted to the scope of the domain of study of the end-user, with the inputs, parameters and outputs he needs.

The process to adapt MeCoSim to each scenario requires the definition of a configuration file. The structure of the file is provided to the designer user in order to configurate the custom simulator he wants to generate. After that, the file is processed by MeCoSim, that loads it in an embed database and generates the custom simulator that comply with the information introduced by the designer user. With this simple task done by the designer and without any software development, the end-user will get a custom simulator for his specific domain problem or case study. A change in the original model structure (desired structure of inputs, outputs or parameters) will be reflected in the simulator with the simple change of the configuration file and its reload in MeCoSim. For more information about the GUI configuration process with MeCoSim, see [7]. 


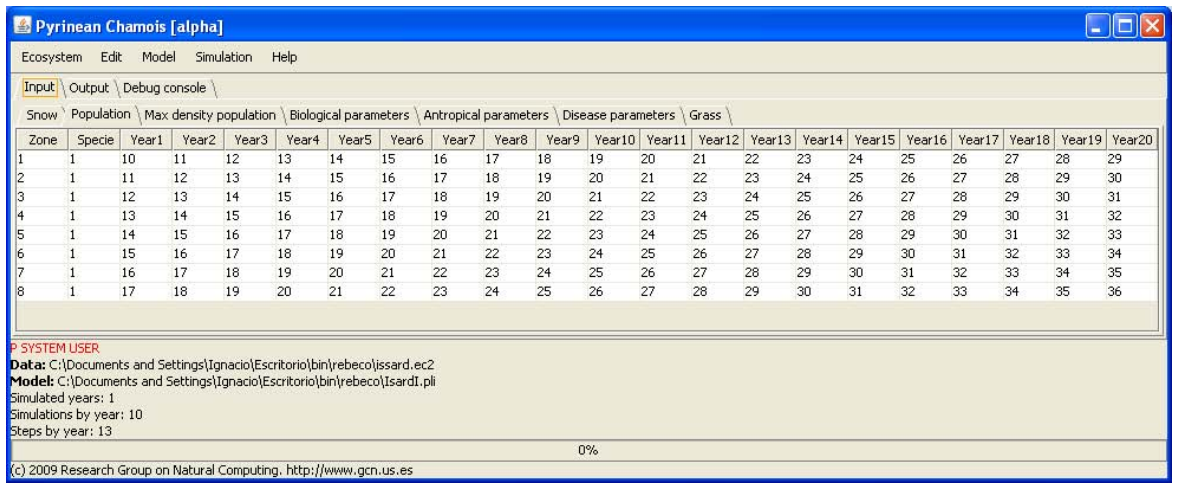

Fig. 3. MeCoSim3: edition process for the initial parameters of the model

Figure 3 shows a snapshot of the software tool MeCoSim with an specific graphic user interface for editing the initial parameters of the model presented in this paper.

\section{Results}

There are experimental data available from 1988 on although censuses where not carried out annually so that, experimental series is not a continuous one. Using the censuses in 1988 as input for the model, 22 years have been simulated repeating the process 50 times for each of the years simulated. Figure 4 shows the results and more specifically, the continuous line represents the average value of the 50 simulations whereas the broken lines correspond to $95 \%$ interval and the dots are the values obtained experimentally. In both Alt Pallars-Aran and Cerdanya-Alt Urgell areas, animals have suffered from pesti-virus infections whereas in Freser-Setcases, the population dynamics have not suffered the disease caused by this infection.

In general, the model behaves well in all cases; nonetheless, there are some experimental values near the lines corresponding to the confidence interval that should be studied.

The model considers the main processes and dynamics of the species although some of them have been omitted because they are considered to be less important. This may explain the differences between the values obtained with the model and experimental ones. Among these factors, it should be highlighted the influence of domestical animals living in the area on the spread of pesti-virus infection. In addition, there are few data regarding the thickness of the snow layer and those used in the model have been obtained from ski resorts so that they may be overdimensioned and then, may affect the results significantly. We suggest studying the relationship between the thickness of the snow layer and other available climatic data in the area such as temperature and the length of the winter interval. 


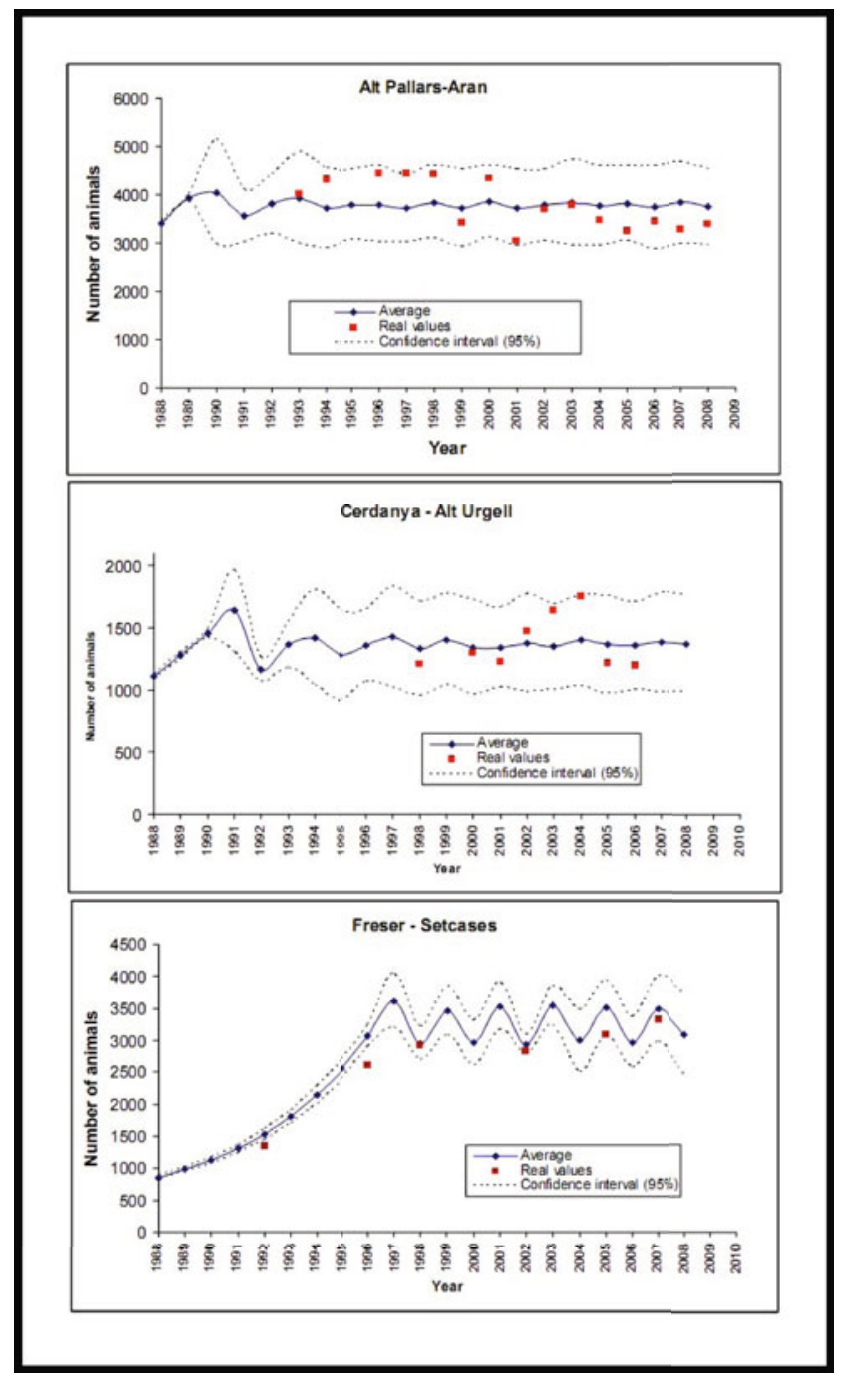

Fig. 4. Results

\section{Conclusions}

This paper presents the first computational model of a real ecosystem from the Catalan Pyrenees involving the Pyrenean Chamois. The model is based on Membrane Computing.

In 2], a general framework for modeling ecosystems using multienvironment $\mathrm{P}$ systems was presented. The rules in that framework were associated with probability functions depending on a number of parameters, such as the simulation time. The model presented in this paper can be considered as a practical 
application of the introduced framework previously. The model is still under the experimental validation phase. Some preliminary validation of this model has been assessed by ecologists resulting in very encouraging and promising results that will be reported elsewhere. In order to assist the validation of the model, we are developing a simulation tool called MeCoSim [7. This software allows the designer user to specify the Graphics User Interface for a specific model by editing a configuration file.

\section{References}

1. Cardona, M., Colomer, M.A., Pérez-Jiménez, M.J., Sanuy, D., Margalida, A.: Modelling ecosystems using P Systems: The Bearded Vulture, a case of study. In: Corne, D.W., Frisco, P., Păun, G., Rozenberg, G., Salomaa, A. (eds.) WMC 2008. LNCS, vol. 5391, pp. 137-156. Springer, Heidelberg (2009)

2. Cardona, M., Colomer, M.A., Margalida, A., Palau, A., Pérez-Hurtado, I., PérezJiménez, M.J., Sanuy, D.: A Computational Modeling for real Ecosystems Based on P Systems. Natural Computing, http://dx.doi.org/10.1007/s11047-010-9191-3

3. Colomer, M.A., Margalida, A., Sanuy, D., Pérez-Jiménez, M.J.: A bio-inspired computing model as a new tool for modeling ecosystems: the avian scavengers as a case study. Ecological modelling (in press, 2010)

4. Crampe, J.P., Gaillard, J.M., Loison, A.: L'enneigement hivernal: un facteur de variation du recrutement chez l'isard (Rupicapra pyrenaica pyrenaica). Canadian Journal of Zoology 80, 306-1312 (2002)

5. García-Quismondo, M., Gutiérrez-Escudero, R., Pérez-Hurtado, I., Pérez-Jiménez, M.J., Riscos-Núñez, A.: An overview of P-lingua 2.0. In: Păun, G., Pérez-Jiménez, M.J., Riscos-Núñez, A., Rozenberg, G., Salomaa, A. (eds.) WMC 2009. LNCS, vol. 5957, pp. 264-288. Springer, Heidelberg (2010)

6. Marco, I., Rosell, R., Cabezón, O., Mentaberre, G., Casas, E., Velarde, R., Lavín, S.: Border disease virus among chamois, Spain. Emerging Infectious Diseases 15, 448-451 (2009)

7. Valencia-Cabrera, L., Pérez-Hurtado, I., Pérez-Jiménez, M.J., Colomer, M.A.: MecoSim: A General purpose software tool for simulating biological phenomena by means of $\mathrm{P}$ systems. In: Pre-proceedings of the IEEE Fifth International Conference on Bio-Inspired Computing: Theories and Applications (BIC-TA 2010) (in press, 2010)

8. GPL license, http://www.gnu.org/copyleft/gpl.html

9. The P-Lingua website, http://www.p-lingua.org/

10. The pLinguaCore library website, http://www.p-lingua.org/wiki/index.php/PLinguaCore 